Journal of Magnetism and Magnetic Materials 442 (2017) pp. 397-402

\title{
Graphite structure and magnetic parameters of flake graphite cast iron
}

\author{
G. Vértesy ${ }^{1}$, T. Uchimoto ${ }^{2}$, T. Takagi ${ }^{2}$, I. Tomáś $^{3}$, H. Kage $^{4}$ \\ ${ }^{1}$ Hungarian Academy of Sciences, Centre for Energy Research, Institute of Technical Physics and \\ Materials Science, H-1121 Budapest, Konkoly Thege Miklos ut 29-33, Hungary, E-mail: \\ vertesy.gabor@energia.mta.hu \\ ${ }^{2}$ Institute of Fluid Science, Tohoku University, 2-1-1 Katahira, Aoba-ku, Sendai, Miyagi 980- \\ 8577, Japan, E-mails: uchimoto@ ifs.tohoku.ac.jp and takagi@ifs.tohoku.ac.jp \\ ${ }^{3}$ Institute of Physics ASCR, Na Slovance 2, Prague 18221, Czech Republic, E-mail: \\ tomas@fzu.cz \\ ${ }^{4}$ Kusaka Rare Metal Co. Ltd., Hitotsumatsu Bidg.No. 3-15, Shiba Daimon 2-chome, Minato-ku, \\ Tokyo, 05-0012 Japan, E-mail: hidehiko-kage@kc-kusaka.co.jp
}

\begin{abstract}
Different matrix and graphite morphologies were generated by a special heat treatment in three chemically different series of flake graphite cast iron samples. As cast, furnace cooled and air cooled samples were investigated. The length of graphite particles and the pearlite volume of samples were determined by metallographic examination and these parameters were compared with the nondestructively measured magnetic parameters. Magnetic measurements were performed by the method of Magnetic Adaptive Testing, which is based on systematic measurement and evaluation of minor magnetic hysteresis loops. It was shown that linear correlation existed between the magnetic quantities and the graphite length, and also between the magnetic quantities and the relative pearlite content in the investigated cast iron. A numerical expression was also determined between magnetic descriptors and relative pearlite content, which does not depend on the detailed experimental conditions.
\end{abstract}

Keywords-Magnetic NDE, Magnetic adaptive testing, Cast iron, Graphite structure, Pearlite content 


\section{INTRODUCTION}

Cast iron is a very important and traditionally used material in industry [1]. The material properties are determined by the structure of the material: metallic matrix composition and graphite morphology. Both of them have a great influence on mechanical characteristics of the construction materials made of cast iron. This is the reason, why the study of both matrix composition of cast iron material (pearlite, ferrite, chill, carbide content) and graphite morphology is important. There are several types of cast iron. In our work we deal with the study of the so-called flake graphite cast iron. This material has good damping properties, which justifies, why flake graphite cast iron is used as brake disk in car industry. The material properties of flake graphite cast iron was studied in [2]. It was shown that both conductivity and permeability depended on graphite morphology.

Nondestructive characterization of the graphite morphology and matrix structure is very important. Several methods exist for this purpose and many publications can be found in the literature in this area. Magnetic permeability, measured by a $B H$ loop tracer seems to be suitable for nondestructive evaluation of graphite structure in flake graphite cast iron. As it was shown in [2], the permeability of the flake graphite cast iron materials depends mainly on the graphite structure rather than on the matrix. However, the permeability changes slightly according to the heat treatment procedure. Another method, the magnetic incremental permeability method [3] is also known as nondestructive magnetic method for studying material behaviour. This method was applied to a set of cast iron samples [4]. This study aimed to quantitatively evaluate the chill structure in ductile cast iron using this method. The relationship between some parameters of the incremental permeability method and the micro-structure of the matrix (ferrite, pearlite, chill) was discussed.

As a promising new way, we have investigated recently, how a magnetic hysteresis method, called Magnetic Adaptive Testing (MAT) can be applied for the investigation of cast iron properties. MAT is based on systematic measurement and evaluation of minor magnetic hysteresis loops. A review of this method can be found in [5], and details of magnetic parameters evaluation are also given detailed.

Different nondestructive magnetic methods - major hysteresis loop measurement, Barkhausen noise measurement and MAT - were applied on a plastically deformed series of transformation induced plasticity (TRIP) steel samples [6]. The results of these methods were compared with each other and good correlation was found between magnetic characteristics, measured in different ways, but MAT was found as the most sensitive method.

By applying MAT, a measurement series was performed on three flake graphite as-cast samples. All investigated samples were in as-cast condition, and their chemical composition was different. An excellent linear correlation was demonstrated between magnetic parameters and graphite morphology [7].

The purpose of the present work is to study similar correlation on three other series of flake graphite cast iron samples, where apart from the as-cast (AS) samples (which typically have a mixed ferrite-pearlite matrix) two kinds of heat treatments (annealing (AN) to obtain a mainly ferrite based matrix and normalization (NR) to obtain a mainly pearlite-based matrix) were performed. We are going also to find correlation between the MAT parameters and the pearlite relative content of the metal matrices in these new series of samples. In this way the present work is a direct continuation of [7] on the three extended series of flake graphite cast iron samples. We 
assumed, and tried to manifest it by our measurements, that the heat treatment modifies the graphite structure and this is well reflected in the magnetic properties.

\section{SAMPLE PREPARATION}

Sample preparation was described detailed in [2]. The chemical composition of the samples are given in Table 1. "CE" means the carbon equivalent of the samples. Their carbon equivalent (CE) values were defined by:

$$
C E=\operatorname{mass} \% \mathrm{C}+\frac{1}{3}(\operatorname{mass} \% \mathrm{Si}+\operatorname{mass} \% \mathrm{P})
$$

and were controlled to produce various graphite shapes and sizes.

Two kinds of heat treatment were performed: annealing, by which a ferrite based matrix was prepared and normalization, by which a pearlite-based matrix was prepared. Six samples were kept at $850^{\circ} \mathrm{C}$ temperature through one hour, and then three samples were cooled down in the furnace for annealing, and the three other samples were cooled down in air atmosphere for normalization. We had also three as cast samples. Such a way 3 as-cast (AS), 3 annealed (AN) and 3 normalized (NR) flake graphite cast iron materials were available for our investigations, which had different matrix structure and different graphite morphology. Hardness measurements were also performed and the results showed that the furnace-cooling and air-cooling annealing produced ferritic and pearlitic matrices.

Table 1. Chemical composition of the flake graphite cast iron samples (values in wt\%)

\begin{tabular}{|c|c|c|c|c|c|c|c|}
\hline \multirow{2}{*}{ Sample } & \multicolumn{7}{|c|}{ Chemical composition } \\
\cline { 2 - 8 } & $\mathbf{C}$ & $\mathbf{S i}$ & $\mathbf{M n}$ & $\mathbf{P}$ & $\mathbf{S}$ & $\mathbf{C r}$ & $\mathbf{T}$ \\
\hline CE4.7 & 3.77 & 2.78 & 0.78 & 0.025 & 0.015 & 0.029 & 0.015 \\
\hline CE4.1 & 3.36 & 2.15 & 0.69 & 0.018 & 0.010 & 0.014 & 0.011 \\
\hline CE3.7 & 3.13 & 1.66 & 0.72 & 0.017 & 0.020 & 0.038 & 0.010 \\
\hline
\end{tabular}

The samples were investigated by metallographic examination. The features of graphite structure is also described detailed in [2]. An image processing software was used for determination of graphite morphology. Fig. 1 shows the structure of the 9 investigated samples.

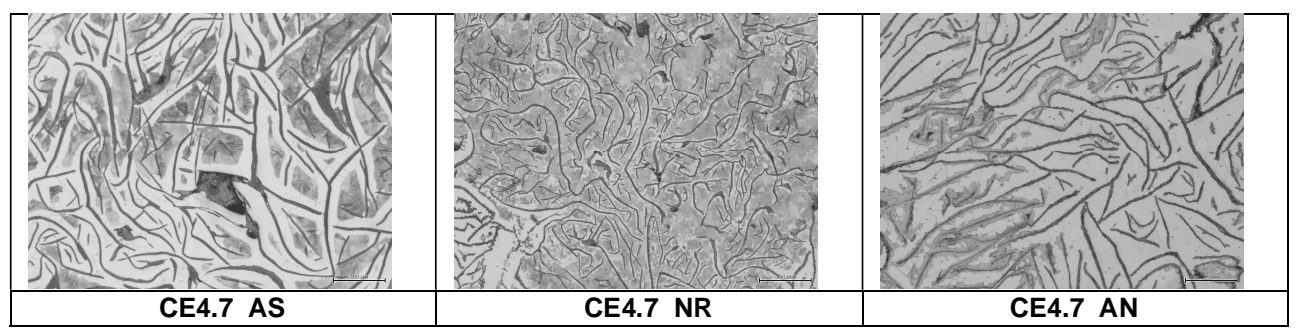




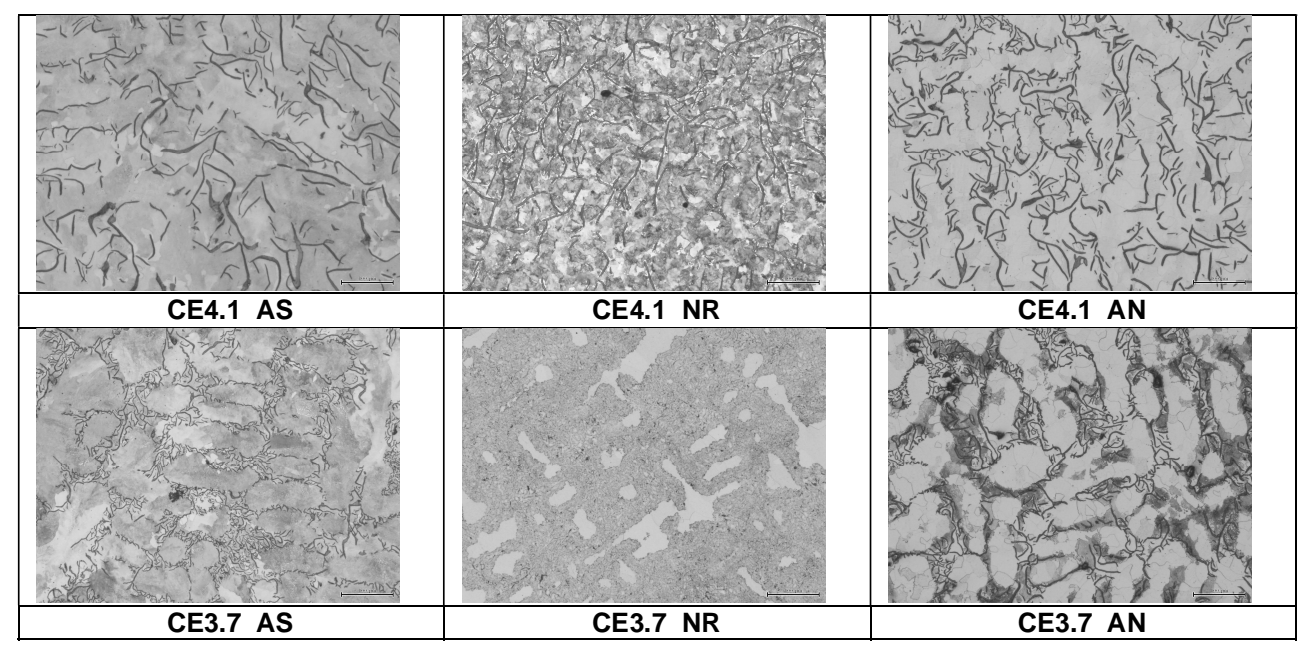

Fig. 1. Microphotographs of the three investigated materials, each having different heat treatments. The scale, which is given in the right bottom corner of the photos, represents $200 \mu \mathrm{m}$ length.

The pearlite ratio and the graphite length of the graphite flakes are shown in Table 2 for all the investigated samples. CE3.7NR has a problem. Due to the normalizing process, the graphite decreased considerably and we observed this sort of phenomenon. NA means we could not carried out microstructure analysis because of the above reason.

Table 2. Pearlite ratio and graphite length of the investigated samples

\begin{tabular}{|l|l|c|c|}
\hline Sample & Thermal treatment & $\begin{array}{c}\text { Relative pearlite } \\
\text { volume, } \mathbf{P}\end{array}$ & $\begin{array}{c}\text { Graphite length } \\
(\boldsymbol{\mu} \mathbf{m})\end{array}$ \\
\hline CE4.7 & as cast (AS) & 0,494 & 58,5 \\
\hline CE4.7 & furnace cooling(AN) & 0,235 & 67,2 \\
\hline CE4.7 & air cooling(NR) & 0,734 & 47,5 \\
\hline CE4.1 & as cast (AS) & 0,859 & 40,4 \\
\hline CE4.1 & furnace cooling(AN) & 0,115 & 37,8 \\
\hline CE4.1 & air cooling(NR) & 0,912 & 41,2 \\
\hline CE3.7 & as cast (AS) & 0,714 & 28,8 \\
\hline CE3.7 & furnace cooling(AN) & 0,411 & 32,9 \\
\hline CE3.7 & air cooling(NR) & NA & NA \\
\hline
\end{tabular}

The investigated samples were half of a $50 \mathrm{~mm}$ diameter disk and they were $12 \mathrm{~mm}$ thick, with smooth surface.

\section{MAGNETIC ADAPTIVE TESTING}

Magnetic adaptive testing was used for magnetic measurements. Series of minor magnetic hysteresis loops were measured by using a yoke for magnetizing the samples. The material of the yoke - used for magnetization of samples -- was laminated Fe-Si transformer core. The size of the yoke was determined to correspond to the size and shape of samples. Exciting coil (200 turns) and sensing coil (75 turns) were directly wound on the yoke legs. 
During MAT process permeability parameters, $\mu_{i j}$-descriptors, are calculated, coordinates of which gives the magnetic field value, $h_{a i}$, on the minor loop with amplitude $h_{b j}$. Each element of the matrix, obtained on the actual sample is normalized by the corresponding element of the reference sample. Such a way the normalized $\mu_{i j}$-descriptors holds information about the changes, which happened in the deformed/degraded/processed samples.

In our case it is not possible to determine the exact value of magnetic field inside the sample, due to the existing air gap between the yoke's leg and sample surface. Because of this the magnetizing current, $h_{a}$, (given in $\mathrm{mA}$ ) is used to characterize the samples' magnetization when MAT descriptors $\mu_{=} \mu\left(h_{a i}, h_{b i}\right)$ are calculated. The real value of magnetic field inside the sample is always proportional with the magnetizing current at a given arrangement, so this simplification does not cause any problem in the calculated (normalized) MAT descriptors. The surface quality of the samples and the air gap for all samples are the same. It means that the correlation between magnetizing current and internal field is for all samples is really comparable.

The magnetizing current rate of change was $2.7 \mathrm{~A} / \mathrm{s}$ in all measurements. However, the value of this parameter is not very important, because, as it was shown in [8] the speed of magnetization has an influence on the sensitivity of the magnetic parameters as function of the given independent parameter, but the type of correlation is never modified.

Another feature of Magnetic Adaptive Testing is, that the sensitivity of MAT descriptors with respect to the independent variable of the measured material can also be calculated. This "sensitivity map" yields important information about the modification of the investigated magnetic descriptor as a function of the independent characteristics. Sensitivity map also shows, how reliable the MAT descriptors can be determined. Large plateaus indicate reliable points, where descriptors depend only very slightly from the actual choice of the exact $h_{a i}$ and $h_{b j}$ values.

\section{RESULTS AND DISCUSSION}

\section{Pearlite content}

During the samples' preparation annealing and normalization were carried out in order to control the relative volumes of pearlite and ferrite in the metal matrix. As the metal matrix is composed from those two constituents only, denoting $P$ the relative volume of pearlite and $F$ that of ferrite, evidently always $P+F=1$. Values of $P$ were determined from microphotographs similar to those in Fig.1, using an image processing software and the measured $P$-values are shown in Table 1. MAT measurements were performed on each sample and the $\mu$-degradation functions were evaluated with $P$ as the independent parameter. The resulting dependence of the top sensitive MAT descriptor, $\mu_{t o p}=\mu\left(h_{a}=300 \mathrm{~mA}, h_{b}=1200 \mathrm{~mA}\right)$, is shown in Fig. $2 \mathrm{a}$. 

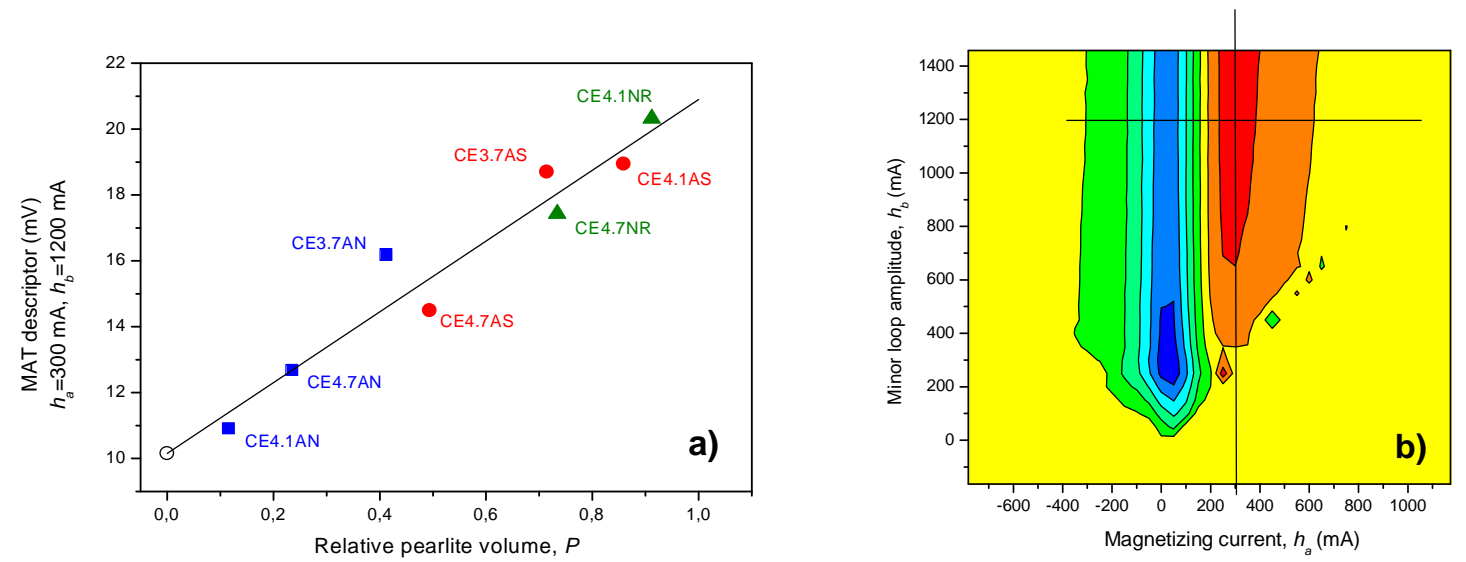

Fig.2 (a) The optimal MAT $\mu_{\text {top }}$-descriptor (not normalized) with coordinates $\mu_{\text {top }}=\mu\left(h_{a}=300 \mathrm{~mA}, h_{b}=1200 \mathrm{~mA}\right)$ versus the relative volume content of pearlite in the matrix, $P$. Hollow circle shows the normalizing constant, which will be used in Fig. 3. (b) The sensitivity map with position of the optimum coordinates shown by the crossing of lines.

All the measured samples are taken into account within Fig. 2a and all the not normalized descriptor points are lying very close to a linear fit. The fitting straight line determines the most probable value of the $\mu_{\text {top }}$ for a $P=0$ sample (i.e. a not measured and presently not existing sample with completely ferritic matrix) to be $\mu_{\text {top }}(P=0)=10.15 \mathrm{mV}$. The numerical values of the $\mu_{\text {top }}$ descriptor evidently depend on the configuration of the experiment (sample dimensions, yoke dimensions, yoke windings, etc.). If, however, values of the $\mu_{\text {top }}$ descriptor are normalized by its value $\mu_{\text {top }}(P=0)$, we get a normalized plot, which does not depend on the detailed experimental conditions any more, and where the best-fit straight line (after interchanging the vertical and horizontal axes) can be generally applied for determination of the $P$ (and/or $F$ ) values in any cast iron with the two-components metal matrix made up from the pearlite and ferrite only. The resulting graph is shown in Fig. 3 and the best-fit straight line is given by the simple equation

$$
P=\mu_{\text {top norm }}(P)-1
$$

The physical content of Fig. 2a and Fig. 3 is the same, however, Fig. 3 represents better the meaning of Eq. 1, and shows the estimated value of $P$ for sample CE3.7NR. In Eq.(1) the $\mu_{\text {top norm }}(P)$ are the normalized values of the top sensitive of the MAT degradation functions, $\mu_{\text {top }}(P)$, normalized by the value of $\mu_{\text {top }}(P=0)$, i.e by the $\mu_{\text {top }}$-descriptor measured for the sample with a fully ferritic matrix. If, similarly as in our case, the $P=0$ sample is not available, it is possible to determine the normalizing $\mu_{\text {top }}(P=0)$ value from at least two samples with independently measured $P$ (and/or $F$ ) values. 


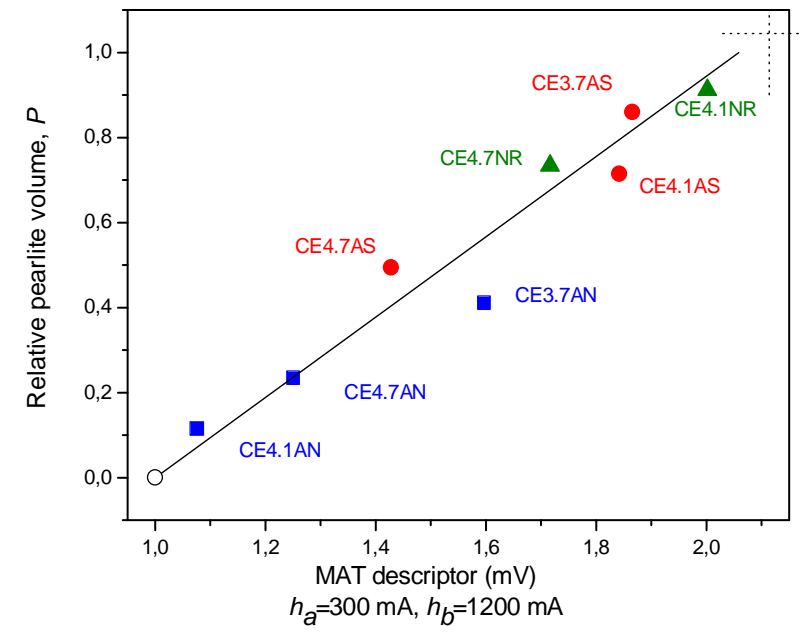

Fig. 3. Plot of the relative volume content of pearlite in the matrix, $P$, versus the normalized MAT descriptor $\mu_{\text {top norm }}\left(h_{a}=300 \mathrm{~mA}, h_{b}=1200 \mathrm{~mA}\right)$. The point marked by a hollow circle is not a measured point, it was used only for normalization. The vertical dot line shows the measured

MAT descriptor of sample CE3.7NR, and its crossing with the linear fit, determines the corresponding $P$ value.

It is expedient to mention here, that the $\mu_{\text {top }}$ descriptor in the MAT measurement is not any singular value measurable at the current coordinates $h_{a}=300 \mathrm{~mA}, h_{b}=1200 \mathrm{~mA}$ only, but - as it is shown in the map of sensitivity of the available degradation functions, see Fig. $2 \mathrm{~b}$ - the same $\mu_{\text {top }}$ magnitudes can be obtained from measurements at $h_{a}=300 \mathrm{~mA}$ with any minor loop amplitudes $h_{b} \geq 500 \mathrm{~mA}$.

It was assumed from the way of preparation that CE3.7NR has a completely pearlitic matrix, but we could not determine the value of $P$ from microphotographs (see Table 3 ). However, this sample was also measured by the magnetic method, and using the $\mu_{\text {top norm }}\left(h_{a}=300 \mathrm{~mA}, h_{b}=1200 \mathrm{~mA}\right)$ value of this sample and Equation (1), we can determine $P$ from $\mu_{\text {top norm }}$ value. This $\mu_{\text {top norm }}$ value of sample CE3.7NR is 2.12 , and the corresponding $P$ is 1.04. Graphically it is shown in Fig. 3 by the crossing of the dashed lines. The estimated value of $P$ is very close (within the experimental error) to the expected $P=1$.

It a very strong argument, that the relative pearlite content can be nondestructively determined by magnetic measurements with good reliability, regardless of the actual sample and regardless of the applied parameters of the concrete MAT measurement (type of yoke, windings, etc.). This fact becomes even more remarkable if we take into account relationships between graphite lengths and pearlite contents, which correlate with each other very well within the same series of samples (the same chemical composition), but it is very different for the three series of samples, as presented in Fig. 4. Fig. 4 indicates that the three investigated sample series have rather different behaviour, but nevertheless, the properly chosen MAT descriptor characterize the pearlite content rather well, regardless on the individual samples. 


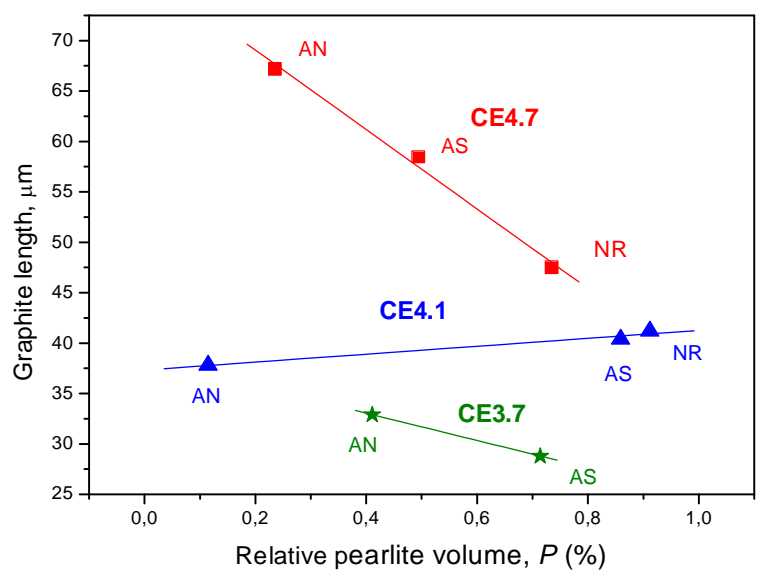

Fig. 4. Graphite length as a function of the pearlite content for the three investigated sample series.

\section{Graphite length}

In view of any technical application, graphite length is a very important parameter of the flake graphite cast iron, perhaps even more important than the pearlite content. Looking at Fig. 4 it is evident that the pearlite content affects the relationship between graphite length and MAT descriptors. Because of this, MAT descriptors were evaluated also as functions of the graphite length. The optimal MAT $\mu$-degradation functions were considered again as functions of a given independent parameter, this time, however, of the length of the graphite flakes. The correlation between the magnetic parameters and the graphite length can be seen in Fig. 5 for all the three series of differently thermally treated samples. (In case of the normalized sample CE3.7NR decarburization occurred, any graphite structure disappeared, as can be seen in Fig. 1 and therefore the sample is missing in Fig. 5a).
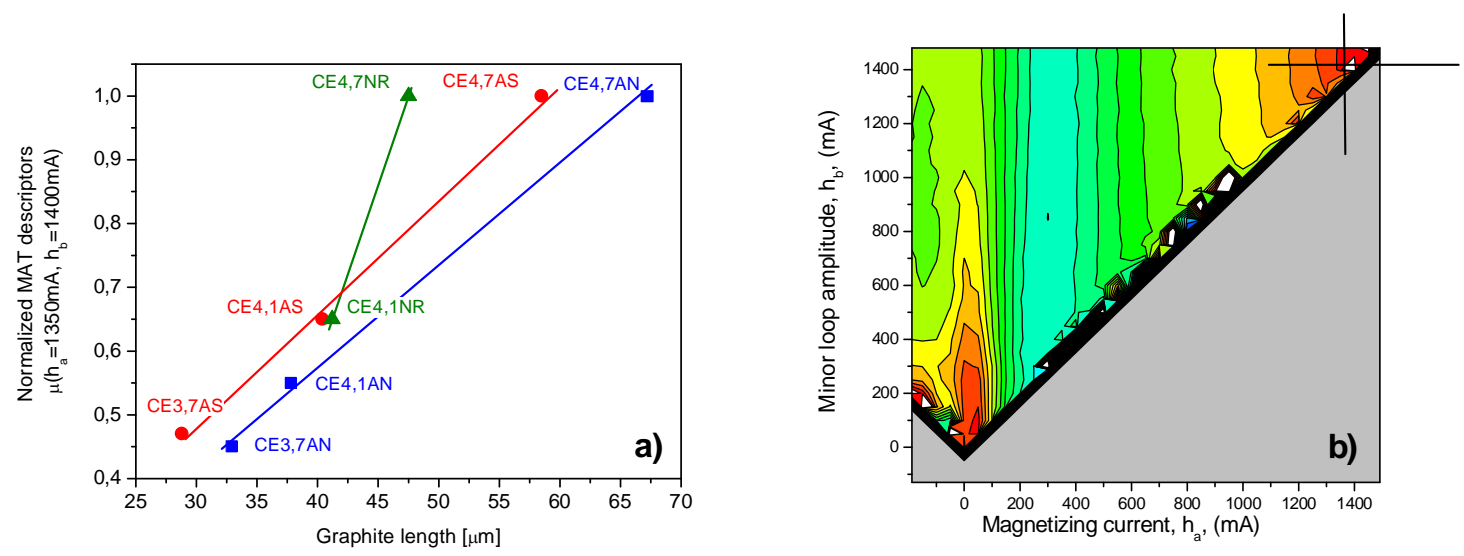
Fig. 5 (a) The optimally chosen normalized MAT descriptor $\mu\left(h_{a}=1350 \mathrm{~mA}, h_{b}=1400 \mathrm{~mA}\right)$ for the as-cast, annealed, and normalized samples. (b) Sensitivity map corresponding to the $\mu$ degradation functions of the as-cast samples depending on the graphite length. The crossing lines show coordinates of the optimum descriptor.

The sensitivity map corresponding to the as-cast samples, showing why just the $\mu$ degradation function with coordinates $\left(h_{a}=1350 \mathrm{~mA}, h_{b}=1400 \mathrm{~mA}\right)$ was chosen as the optimal MAT descriptor of Fig. 5a, can be seen in Fig. 5b. The crossing lines are situated in the most sensitive (red) area, and the red area is large enough to ensure good reproducibility of the measurement, as nearly the same results are obtained for the whole interval of coordinates $\left(1250 \leq h_{a} \leq 1450 \mathrm{~mA}, 1300 \leq h_{b} \leq 1500 \mathrm{~mA}\right)$. An almost identical sensitivity map is obtained for the annealed and normalized samples.

As shown in Fig. 5a, very regular linear correlation was found between the length of the graphite flakes and the magnetic parameters in the three as-cast samples having different chemical compositions. This correlation is identical with that presented in [6]. It turned out from the present experiments, however, that the graphite structure changes by any heat treatment, and therefore similar correlations are shown in Fig. 5a also for the annealed and normalized samples. Remarkably similar slopes are observed for the as-cast and the annealed samples. The normalized samples look different. Due to the only two measured points in the last case we do not dare to draw any sure conclusion, but the different slope is evident.

The previous graphs show the correlation between MAT descriptors and graphite length if the same type of heat treatment is applied for the samples having different chemical composition. However, the systematic analysis of magnetic parameters makes also possible to investigate how the parameters change within the same chemical composition, if the annealing conditions are different. The results are shown in Fig.6.
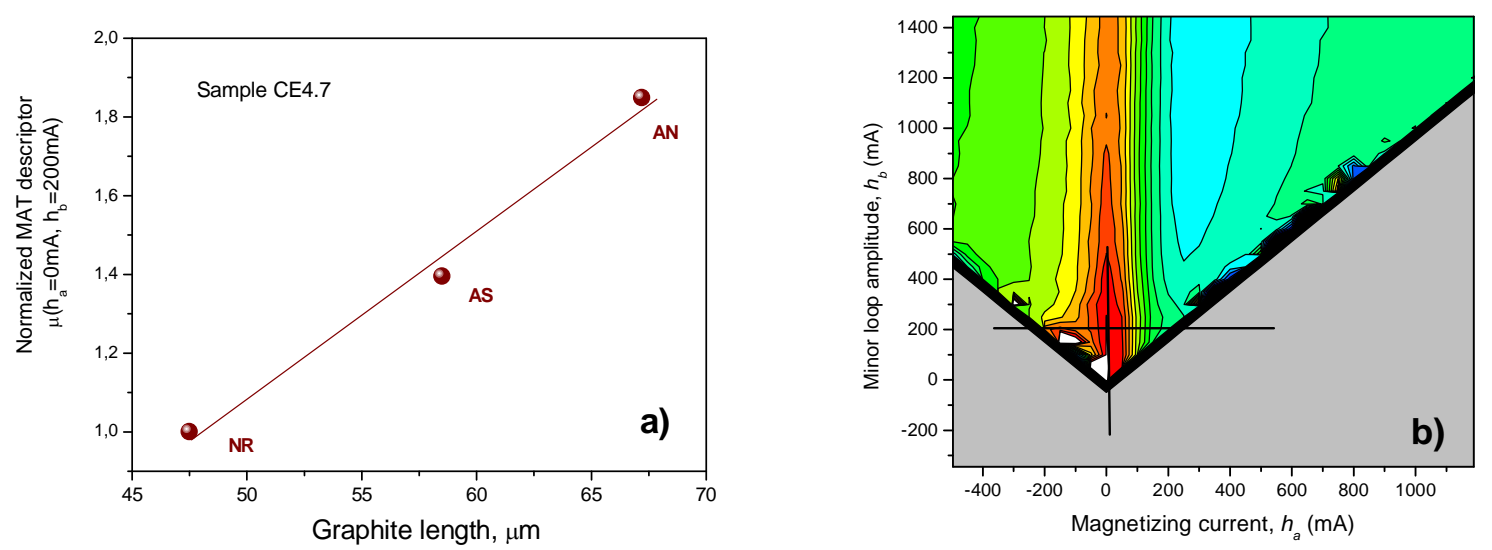

Fig 6. The optimally chosen MAT descriptors for CE4.7 samples (a) and the corresponding sensitivity map (b).

It is worth of mentioning that in this case (samples CE4.7 at different heat treatments) different MAT descriptors give the best correlation, than it was experienced in Fig. 5. Here MAT 
descriptors $\left(h_{a}=0 \mathrm{~mA}, h_{b}=200 \mathrm{~mA}\right)$ characterize the best the correlation between magnetic parameters and graphite morphology. The corresponding sensitivity map indicates as well, that the red area is large enough to ensure good reproducibility of the measurement.

\section{CONCLUSIONS}

By applying Magnetic Adaptive Testing on flake graphite cast iron samples, having different matrix structure and graphite morphology, linear correlation was found between graphite length and MAT descriptors. This correlation is valid if samples with different chemical composition but the same heat treatment, and also if samples with different heat treatments but same chemical composition are considered.

Similarly good correlation was found between pearlite content in the metal matrix and MAT descriptors regardless that these parameters do not correlate with each other if we consider all the three series of samples.

Numerical expression was determined between magnetic characteristics and relative pearlite content, which does not depend on the detailed experimental conditions any more. This expression can be generally applied for determination of the pearlite values in any cast iron with the two-components metal matrix made up from the pearlite and ferrite only.

Different MAT descriptors were used to characterize the correlation with pearlite ratio, and to characterize the correlation with graphite length. This fact shows very well the multiparametric behaviour of MAT: from the big data pool we pick up those descriptors, which characterize the best correlation with a given independent parameter.

\section{ACKNOWLEDGMENTS}

This work was supported by the Hungarian Scientific Research Fund (project K 111662), by the Researcher Exchange Program between the Japan Society for Promotion of Science and Hungarian Academy of Sciences, and by the Researcher Exchange Program between the Czech Academy of Sciences and Hungarian Academy of Sciences and partially also by the JSPS Coreto-Core Program (Advanced Research Networks: International research core on smart layered materials and structures for energy saving). One of the co-authors (I.T.) appreciates support of the project No.14-36566G of the Czech Science Foundation.

\section{REFERENCES}

[1] C.F. Walton and T.J. Opar (Eds.), Iron casting handbook, Iron Casting Society, Inc., New York, 1981.

[2] T. Uchimoto, T. Takagi, T. Abe, Electromagnetic nondestructive evaluation of graphite structures in flake graphite cast iron, Materials Transactions, 51 (2010) 1114-1119

[3] A.Yashan, Measurements and semi-analytical modeling of incremental permeability using eddy current coil in the presence of magnetic hysteresis, Electromagnetic Nondestructive Evaluation (VI), IOS press, (2002), 150-157.

[4] T. Matsumoto, T. Uchimoto, T. Takagi, G. Vértesy, Evaluation of Chill Structure in Ductile Cast Iron by Incremental Permeability Method, International Journal of Applied Electromagnetics and Mechanics, 52 (2016) 1599-1605 
[5] I.Tomáš, G.Vértesy, „Magnetic Adaptive Testing“, in Nondestructive Testing Methods and New Applications, M.Omar (Ed.), ISBN: 978-953-51-0108-6, (2012), InTech: http://www.intechopen.com/articles/show/title/magnetic-adaptive-testing

[6] G. Vértesy, I. Mészáros, I. Tomáš, Nondestructive magnetic characterization of TRIP steels, NDT \& E INTERNATIONAL, 54 (2013) 107-114

[7] G. Vértesy, T. Uchimoto, T. Takagi, I. Tomáš, Flake graphite cast iron investigated by a magnetic method, IEEE Trans. Magn, Vol. 50. No. 4, April 2014, 6200404

[8] I. Tomáš, G. Vértesy, J. Kadlecová, J. Magn. Magn. Mater, 321 (2009) 1019-1024 